\title{
Electrophoresis in a non-Newtonian fluid: sphere in a spherical cavity
}

\author{
Eric Lee, Yu-Fen Huang, and Jyh-Ping Hsu* \\ Department of Chemical Engineering, National Taiwan University, Taipei, Taiwan 10617, Republic of China
}

Received 29 May 2002; accepted 4 October 2002

\begin{abstract}
The electrophoretic behavior of a sphere in a non-Newtonian fluid is investigated theoretically by analyzing the phenomenon that occurs in a spherical cavity under the condition of a weak applied electrical field. Non-Newtonian behavior in the liquid phase may be due to, for example, the addition of polymer to a colloidal dispersion to improve its stability. It may also arise from the increase in the volume fraction of the dispersed phase such as the slurry used in chemical mechanical polishing. A Carreau model is adopted to characterize the shear-thinning behavior of the liquid phase. We show that the difference between the mobility of the particle based on the present model and that based on the corresponding Newtonian fluid increases with the decrease in the thickness of a double layer. The shear-thinning nature of the liquid phase has the effect of increasing the mobility.
\end{abstract}

(C) 2003 Elsevier Science (USA). All rights reserved.

Keywords: Electrophoresis; Sphere; Spherical cavity; Carreau fluid

\section{Introduction}

The surface properties of a charged entity are often estimated by an electrophoresis measurement. Its theoretical basis was founded by Smoluchowski [1] who showed that the electrophoretic mobility of a particle, $\mu_{E}$, defined by the ratio $U / E, U$ and $E$ being respectively the magnitude of the electrophoretic velocity and the strength of the applied electric field, and its zeta potential, $\zeta$, are related by

$\mu_{E}=\frac{\varepsilon_{r} \varepsilon_{0} \zeta}{\eta}$

where $\varepsilon_{r}$ and $\eta$ are respectively the relative permittivity and the viscosity of the liquid phase and $\varepsilon_{0}$ is the permittivity of a vacuum. Equation (1) provides a concise, yet clear, quantitative description for the behavior of a charged entity in an applied electric field and is widely used in various areas in practice. It should be pointed out, however, that its derivation was based on the assumptions of a rigid, nonconductive entity at low surface potentials, weak applied electric field, thin double layer, negligible double-layer polarization and boundary effect, and Newtonian fluid. Various attempts have been made to extend the original analysis of Smoluchowski by relaxing some of these assumptions.

\footnotetext{
* Corresponding author.

E-mail address: jphsu@ccms.ntu.edu.tw (J.-P. Hsu).
}

The electrophoretic phenomenon is described by the socalled electrokinetic equations, which include the governing equations for the flow, the concentration, and the electric fields. Since these equations are coupled and highly nonlinear, solving them analytically is almost impossible. This difficulty can often be circumvented by considering limiting cases such as simple geometry, very thin or very thick double layers, and low surface potentials [1-8]. In general, a numerical scheme needs to be adopted [9-12]. Adopting a shooting method, O'Brien and White [9] were able to derive the electrophoretic velocity of a sphere in an infinite fluid for the case of arbitrary double-layer thickness and surface potential, taking the effect of double-layer polarization into account. Lee et al. [10-13] discussed the electrophoretic phenomenon of a concentrated spherical dispersion and that of a sphere in a spherical cavity; a pseudo-spectral method was proposed in the numerical procedure. The results are applicable to arbitrary electrical potential and double-layer thickness, mixed-type boundary condition, and double-layer polarization.

Although the electrophoretic phenomenon has been studied extensively, almost all of the available results are for the case where the liquid phase is a Newtonian fluid, and the corresponding result for the case of non-Newtonian fluid is extremely limited. Presumably, this is mainly due to the difficulties involved in solving the electrokinetic equations. Recent studies reveal that the effect of a non-Newtonian 
fluid needs to be considered in some problems, which are of both fundamental and practical significance. For example, the addition of polymer to a colloidal dispersion to improve its stability leads to shear-thinning behavior in the liquid phase [14]. Non-Newtonian behavior may also arise from the increase in the volume fraction of the dispersed phase [15]. The volume fraction of the slurry used in semiconductor processing, for instance, can easily exceed $10 \%$ and assuming Newtonian behavior is unrealistic in this case.

The constitutive equations for a generalized Newtonian fluid (GNF) can be expressed as [16]

$\tau=-\eta(\dot{\gamma}) \dot{\gamma}$

$\dot{\gamma}=\nabla \mathbf{v}+(\nabla \mathbf{v})^{\mathrm{T}}$.

In these expressions, $\boldsymbol{\tau}, \dot{\boldsymbol{\gamma}}, \boldsymbol{\eta}$, and $\mathbf{v}$ are respectively the stress tensor, the rate of strain tensor, the apparent viscosity, and the velocity of the fluid, $\nabla$ is the gradient operator, and the superscript " $T$ " denotes matrix transpose. Note that if the apparent viscosity remains constant, Eqs. (2) and (3) reduce to the constitutive equations for a Newtonian fluid. Shearthinning fluid is one of the most important fluids which exhibit non-Newtonian behavior. Carreau and others [16,17] proposed the following three-parameter correlation relation, the so-called Carreau model, for the description of the behavior of a shear-thinning fluid,

$\eta(\dot{\gamma})=\eta_{0}\left[1+(\lambda \dot{\gamma})^{\beta}\right]^{(n-1) / \beta}$,

where $\eta_{0}$ is the viscosity corresponding to the minimum shear rate, $\lambda$ is the relaxation time constant, $n$ is the powerlaw exponent (since it describes the slope of $\left(\eta-\eta_{\infty}\right) /\left(\eta_{0}-\right.$ $\left.\eta_{\infty}\right)$ in the power-law region), and $\beta$ is a dimensionless parameter that describes the transition region between the zero-shear-rate region and the power-law region. According to the Carreau model, the variation of viscosity as a function of shear rate can be divided into three regions: (a) For small shear rates, the viscosity is insensitive to the variation in the shear rate, the so-called zero-shear-rate viscosity region. (b) If shear rate exceeds a critical value, the viscosity decreases monotonically with the shear rate, the so-called power-law fluid region. (c) If shear rate is large, the viscosity becomes insensitive to the variation in the shear rate again, the so-called infinite-shear-rate viscosity region. Yasuda et al. [18] proposed the following five-parameter CarreauYasuda model:

$\eta(\dot{\gamma})=\eta_{\infty}+\left(\eta_{0}-\eta_{\infty}\right)\left[1+(\lambda \dot{\gamma})^{\beta}\right]^{(n-1) / \beta}$,

where $\eta_{0}$ and $\eta_{\infty}$ are respectively the zero-shear-rate viscosity and infinite-shear-rate viscosity. These two parameters are roughly constant, and their effects are usually insignificant. This five-parameter model has sufficient flexibility to fit a wide variety of experimental $\boldsymbol{\eta}(\dot{\gamma})$ curves. It has proven to be useful for numerical calculations. It has the merit that if either $n \rightarrow 1$ or $\lambda \rightarrow 0$, it reduces to a Newtonian fluid, and it becomes a power-law fluid if $\lambda$ is sufficiently large. For many concentrated polymer solutions, good fit can be obtained by choosing $\beta=2$ and $\eta_{\infty}=0$. In particular, it is capable of describing the characteristics of the melts of many polymeric materials of industrial significance such as high-density polyethylene, low-density polyethylene, and polystyrene.

In the present study the electrophoretic behavior of a particle in a non-Newtonian fluid is analyzed for the case of low electrical potential. In particular, the boundary effect is taken into account by considering a sphere in a spherical cavity.

\section{Theory}

Referring to Fig. 1, we consider the electrophoresis of a rigid sphere of radius $a$ in a spherical cavity of radius $b$ filled with an electrolyte solution. Both the particle and the cavity are nonconductive. Let $H=(a / b)$, which measures the significance of the presence of the cavity. The particle is placed at the center of the cavity, and the space between sphere and cavity is filled with a Carreau (shear-thinning) fluid. The spherical coordinates $(r, \theta, \varphi)$ are chosen with its origin located at the center of the cavity. An electric field $\mathbf{E}$ is applied in the $Z$-direction, the particle moves with velocity $\mathbf{U}$, and the cavity remains stationary toward the laboratory frame of reference. Suppose that $\mathbf{E}$ is weak and the polarization or relaxation of the double layer surrounding the particle is negligible.

\subsection{Electrokinetic equations}

Suppose that the spatial variation in the electrical potential $\phi$ can be described by the Poisson equation,

$\nabla^{2} \phi=-\frac{\rho_{e}}{\varepsilon}$

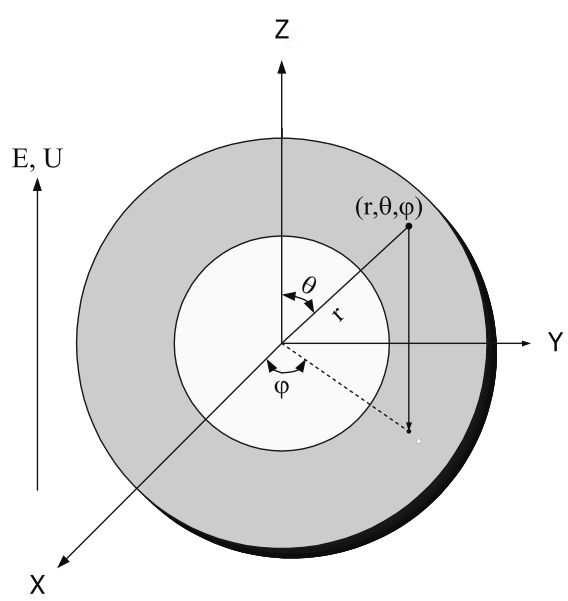

Fig. 1. Schematic representation of the problem considered. A spherical particle of radius $a$ is placed at the center of a spherical cavity of radius $b$. An electrical field $\mathbf{E}$ is applied in the $Z$-direction and $\mathbf{U}$ is the electrophoretic velocity of the particle. The origin of the spherical coordinates $(r, \theta, \varphi)$ coincides with the center of the cavity. 
where $\varepsilon$ is the permittivity of the liquid phase and $\rho_{e}$ is the space charge density, which can be expressed by

$\rho_{e}=\sum_{j=1}^{N} z_{j} e n_{j}$,

where $N$ is the number of ionic species, $z_{j}$ and $n_{j}$ are the valence and the number density of ionic species $j$, and $e$ is the elementary charge.

Suppose that the liquid phase is incompressible and the flow field can be described by the Cauchy momentum equation in the creeping flow regime. Then

$\nabla \cdot \mathbf{v}=0$

$\rho_{f} \frac{\partial \mathbf{v}}{\partial t}=-\nabla \cdot \boldsymbol{\tau}-\nabla p-\rho_{e} \nabla \phi$.

In these expressions $p$ is the pressure and $\mathbf{v}$ and $\rho_{f}$ are respectively the velocity and the density of liquid phase. Note that a body force term, $-\rho_{e} \nabla \phi$, is included in the Cauchy momentum equation to take the effect of electric force into account. The stress tensor $\boldsymbol{\tau}$ in Eq. (9) is described by Eqs. (2) and (3), and the viscosity $\eta$ in Eq. (2) is described by the Carreau model, Eq. (5), with $\beta=2$ and $\eta_{\infty}=0$. Assume the system is at a pseudo-steady state, then the lefthand side of Eq. (9) vanishes. For convenience, the electrical potential is expressed as the sum of the equilibrium potential (or the potential in the absence of the applied electric field), $\phi_{1}$, and a perturbed electrical potential which arises from the applied electric field, $\phi_{2}$. Suppose that the spatial variation in the concentration of ionic species follows the Boltzmann distribution. Then, we have

$$
\begin{aligned}
& \nabla^{2} \phi_{1}=-\frac{\rho_{1}}{\varepsilon}=-\sum_{j=1}^{N} \frac{z_{j} e n_{j 0}}{\varepsilon} \exp \left[-\frac{z_{j} e \phi_{1}}{k_{B} T}\right], \\
& \nabla^{2} \phi_{2}=-\frac{\rho_{2}}{\varepsilon}=-\left[\sum_{j=1}^{N} \frac{z_{j} e n_{j 0}}{\varepsilon} \exp \left[-\frac{z_{j} e\left(\phi_{1}+\phi_{2}\right)}{k_{B} T}\right]\right. \\
& \left.-\sum_{j=1}^{N} \frac{z_{j} e n_{j 0}}{\varepsilon} \exp \left[-\frac{z_{j} e \phi_{1}}{k_{B} T}\right]\right] \text {, } \\
& \phi=\phi_{1}+\phi_{2}, \\
& \rho_{e}=\rho_{1}+\rho_{2} \text {, } \\
& n_{j}=n_{j 0} \exp \left[-\frac{z_{j} e\left(\phi_{1}+\phi_{2}\right)}{k_{B} T}\right] \text {, }
\end{aligned}
$$

where $n_{j 0}$ is the bulk concentration of species $j, k_{B}$ is Boltzmann's constant, and $T$ is the absolute temperature.

The procedure of solving Eqs. (8) and (9) can be simplified by adopting a stream function formulation to reduce the number of variables and equations. Let $\psi$ be the stream function of the flow field under consideration and express the $r$ and the $\theta$ components of the fluid velocity, $\mathbf{v}_{r}$ and $\mathbf{v}_{\theta}$, respectively as $\mathbf{v}_{r}=-\left(1 / r^{2} \sin \theta\right)(\partial \psi / \partial \theta)$ and $\mathbf{v}_{\theta}=(1 / r \sin \theta)(\partial \psi / \partial r)$. These expressions make the incompressibility constraint, Eq. (8), satisfied automatically.
Taking curl on both sides of Eq. (9), the pressure term on its right-hand side can be eliminated, and a governing equation for the stream function, which is coupled with that for the electrical potential, can be obtained. Note that since the apparent viscosity varies with the shear rate, Eq. (9) is nonlinear, and an iterative procedure is necessary.

\subsection{Boundary conditions}

Intuitively, the boundary conditions on a particle surface for the electrical potential depends on the electrostatic model chosen. However, as pointed out by O'Brien and White [9] that if the electrokinetic equations are linearized and the applied field is weak, the electrophoretic mobility becomes independent of the electrostatic boundary conditions chosen on the particle surface. Here, we assume that both the particle and the cavity remain at constant surface potential at equilibrium, characterized respectively by the corresponding zeta potentials, $\zeta_{a}$ and $\zeta_{b}$, respectively. Therefore, the boundary conditions for the equilibrium electrical potential $\phi_{1}$ are

$\phi_{1}=\zeta_{a}, \quad r=a$,

$\phi_{1}=\zeta_{b}, \quad r=b$.

Since the particle is nonconductive, the electric field vanishes on its surface, that is,

$\frac{\partial \phi_{2}}{\partial r}=0, \quad r=a$.

The boundary condition for the perturbed electrical potential on a cavity surface is assumed as

$\frac{\partial \phi_{2}}{\partial r}=-\mathbf{E}_{z} \cos \theta, \quad r=b$.

The particle moves with velocity $\mathbf{U}$ and the cavity is stationary. Therefore, the boundary conditions for liquid velocity are

$\mathbf{v}_{r}=\mathbf{U} \cos \theta, \quad r=a$,

$\mathbf{v}_{\theta}=-\mathbf{U} \sin \theta, \quad r=a$,

$\mathbf{v}_{r}=\mathbf{v}_{\theta}=0, \quad r=b$.

In terms of the stream function we have

$\psi=-\frac{1}{2} U r^{2} \sin \theta, \quad r=a$,

$\frac{\partial \psi}{\partial r}=-U r \sin ^{2} \theta, \quad r=a$,

$\psi=\frac{\partial \psi}{\partial r}=0, \quad r=b$.

The symmetric nature of the problem under consideration requires that

$\frac{\partial \phi_{1}}{\partial \theta}=\frac{\partial \phi_{2}}{\partial \theta}=\psi=\frac{\partial \psi}{\partial \theta}=0, \quad \theta=0$,
$\frac{\partial \phi_{1}}{\partial \theta}=\frac{\partial \phi_{2}}{\partial \theta}=\psi=\frac{\partial \psi}{\partial \theta}=0, \quad \theta=\pi$. 
For a simpler treatment the electrokinetic equations and the associated boundary conditions are rewritten in scaled form. If the liquid phase contains $z_{1}: z_{2}$ electrolyte with $z_{2} / z_{1}=-\alpha$, then $n_{20}=n_{10} / \alpha$. Since $\kappa^{-1}=$ $\left[\varepsilon k_{B} T / \sum n_{j 0}\left(e z_{j}\right)^{2}\right]^{1 / 2}$, we have $n_{10} z_{1}=\left[(\kappa a)^{2} \varepsilon k_{B} T /\right.$ $\left.(1+\alpha) e^{2} a^{2} z_{1}\right]$. The surface potential of particle $\zeta_{a}$ is chosen as the scale parameter for electrical potential, the particle radius $a$ is chosen as the scale parameter for length, and the bulk number concentration of cations $n_{10}$ is chosen as the scale parameter for concentration. We define $\phi_{1}=\phi_{1}^{*} \zeta_{a}$, $\phi_{2}=\phi_{2}^{*} \zeta_{a}, n_{j}=n_{j}^{*} n_{j 0}, r=a r^{*}$, and $\nabla^{*}=a \nabla$. In terms of scaled symbols Eq. (10) becomes

$\nabla^{* 2} \phi_{1}^{*}=-\frac{1}{(1+\alpha)} \frac{(\kappa a)^{2}}{\phi_{r}}\left(n_{1}^{*}-n_{2}^{*}\right)$,

where $n_{1}^{*}=\exp \left(-\phi_{r}\left(\phi_{1}^{*}+\phi_{2}^{*}\right)\right)$ and $n_{2}^{*}=\exp \left(\alpha \phi_{r}\left(\phi_{1}^{*}+\right.\right.$ $\left.\phi_{2}^{*}\right)$ ). The associated boundary conditions are

$\phi_{1}^{*}=1, \quad r^{*}=1$,

$\phi_{1}^{*}=\frac{\zeta_{b}}{\zeta_{a}}, \quad r^{*}=\frac{1}{H}$,

where $\phi_{r}=\zeta_{a} z_{1} e / k_{B} T$. Similarly, the scaled form for Eq. (12) and the associated boundary conditions are

$$
\begin{aligned}
& \nabla^{* 2} \phi_{2}^{*}=-\frac{1}{(1+\alpha)} \frac{(\kappa a)^{2}}{\phi_{r}} \\
& \quad \times\left(\left(n_{1}^{*}-n_{2}^{*}\right)-\left(\exp \left(-\phi_{r} \phi_{1}^{*}\right)-\exp \left(\alpha \phi_{r} \phi_{1}^{*}\right)\right)\right), \\
& \frac{\partial \phi_{2}^{*}}{\partial r^{*}}=0, \quad r^{*}=1, \\
& \frac{\partial \phi_{2}^{*}}{\partial r^{*}}=-\mathbf{E}_{z}^{*} \cos \theta, \quad r^{*}=\frac{1}{H},
\end{aligned}
$$

where $\mathbf{E}_{z}^{*}=\mathbf{E}_{z} a / \zeta_{a}$.
We define the scaled stream function $\psi^{*}$ as $\psi^{*}=$ $\psi / U_{E} a^{2}$, where $U_{E}=\varepsilon \zeta_{a}^{2} / \eta a$ is the electrophoretic velocity evaluated by Smoluchowski's formula when an electric field of strength $\zeta_{a} / a$ is applied. The space charge density $\rho_{e}$ can be expressed as $\rho_{e}=\rho_{0} \rho_{e}^{*}$, where $\rho_{0}=\varepsilon \kappa^{2} \zeta_{a} /(1+\alpha) \phi_{r}$ and $\rho_{e}^{*}=n_{1}^{*}-n_{2}^{*}$. Also, we define $E=E^{*} / a, \eta=\eta^{*} \eta_{0}$, and $\dot{\gamma}=\dot{\gamma}^{*} U_{E} / a$. The scaled form for the stream function for the case when the electrical potential is low is

$$
\begin{gathered}
\eta^{*} E^{* 4} \psi^{*}+\sin \theta\left[\left(\frac{\partial \eta^{*}}{\partial r^{*}} \dot{\gamma}_{r \theta}^{*}+r^{*} \frac{\partial^{2} \eta^{*}}{\partial r^{* 2}} \dot{\gamma}_{r \theta}^{*}+r^{*} \frac{\partial \eta^{*}}{\partial r^{*}} \frac{\partial \dot{\gamma}_{r \theta}^{*}}{\partial r^{*}}\right.\right. \\
\left.+\frac{\partial^{2} \eta^{*}}{\partial r^{*} \partial \theta} \dot{\gamma}_{\theta \theta}^{*}+\frac{\partial \eta^{*}}{\partial \theta} \frac{\partial \dot{\gamma}_{\theta \theta}^{*}}{\partial r^{*}}\right) \\
-\left(\frac{\partial^{2} \eta^{*}}{\partial r^{*} \partial \theta} \dot{\gamma}_{r r}^{*}+\frac{\partial \eta^{*}}{\partial r^{*}} \frac{\partial \dot{\gamma}_{r r}^{*}}{\partial \theta}\right. \\
\left.+\frac{1}{r^{*}} \frac{\partial^{2} \eta^{*}}{\partial \theta^{2}} \dot{\gamma}_{r \theta}^{*}+\frac{1}{r^{*}} \frac{\partial \eta^{*}}{\partial \theta} \frac{\partial \dot{\gamma}_{r \theta}^{*}}{\partial \theta}\right) \\
+\frac{\partial \eta^{*}}{\partial r^{*}}\left(\frac{1}{\sin \theta} \frac{\partial^{3} \psi^{*}}{\partial r^{* 3}}-\frac{\cot \theta}{r^{* 2} \sin \theta} \frac{\partial^{2} \psi^{*}}{\partial r^{*} \partial \theta}\right. \\
+\frac{1}{r^{* 2} \sin \theta} \frac{\partial^{3} \psi^{*}}{\partial r^{*} \partial \theta^{2}}-\frac{2}{r^{* 3} \sin \theta} \frac{\partial^{2} \psi^{*}}{\partial \theta^{2}} \\
\left.+\frac{2 \cot \theta}{r^{* 3} \sin \theta} \frac{\partial \psi^{*}}{\partial \theta}\right) \\
-\frac{\partial \eta^{*}}{\partial \theta}\left(-\frac{1}{r^{* 2} \sin \theta} \frac{\partial^{3} \psi^{*}}{\partial r^{* 2} \partial \theta}-\frac{1}{r^{* 4} \sin \theta} \frac{\partial^{3} \psi^{*}}{\partial \theta^{3}}\right. \\
\left.\left.\quad-\frac{1}{r^{* 4} \sin 3} \frac{\partial \psi^{*}}{\partial \theta}+\frac{\cot \theta}{r^{* 4} \sin \theta} \frac{\partial^{2} \psi^{*}}{\partial \theta^{2}}\right)\right] \\
=-(\kappa a)^{2} \frac{\partial \phi_{1}^{*} \frac{\partial \phi_{2}^{*}}{\partial r^{*}} \sin \theta}{\partial \theta}
\end{gathered}
$$

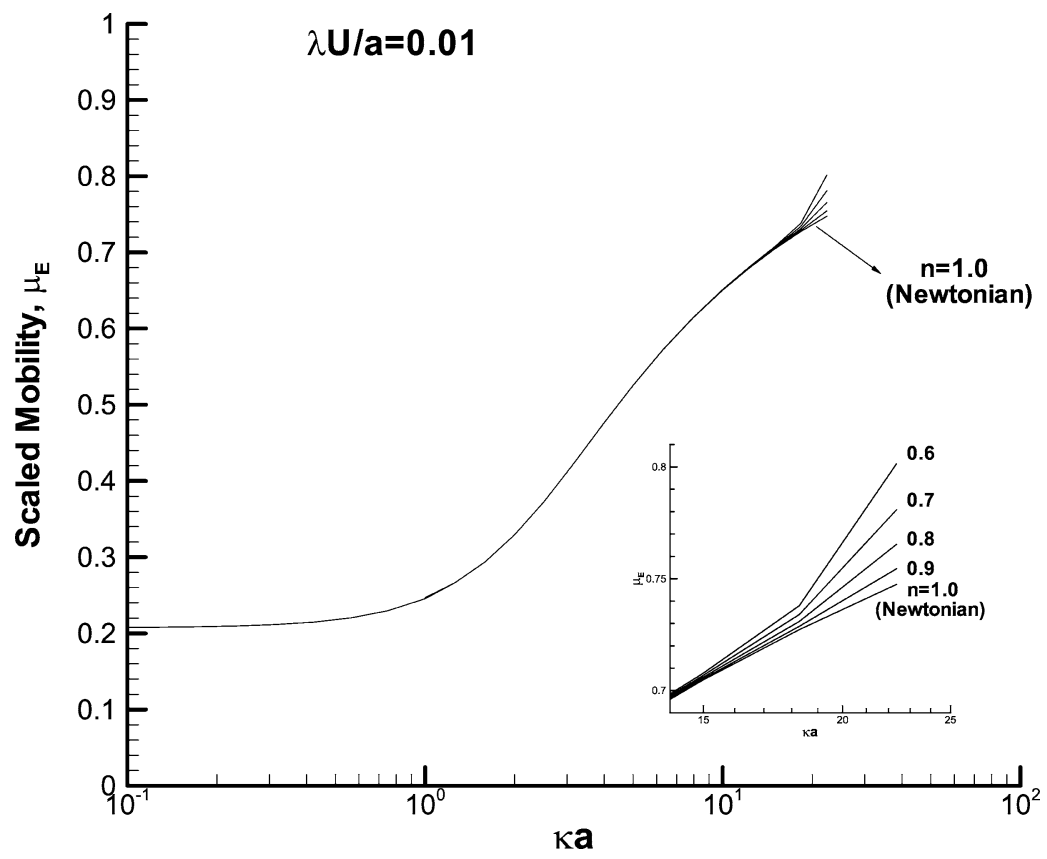

Fig. 2. Variation of scaled mobility $\mu_{E}$ as a function of double-layer thickness $\kappa a$ at various values of parameter $n$ for the case $\lambda U / a=0.01$. 
The associated boundary conditions are

$\psi^{*}=-\frac{1}{2} U^{*} r^{* 2} \sin ^{2} \theta, \quad r^{*}=1$,

$\frac{\partial \psi^{*}}{\partial r^{*}}=-U^{*} r^{*} \sin ^{2} \theta, \quad r^{*}=1$,

$\psi^{*}=\frac{\partial \psi^{*}}{\partial r^{*}}=0, \quad r^{*}=\frac{1}{H}$.

The scaled governing equations and the associated boundary conditions are solved numerically by adopting a pseudospectral method [19] based on Chebyshev polynomials. The physical domain described by the spherical coordinates is mapped onto a square computational domain $[-1,1] \times$ $[-1,1]$. The discretization of the computational domain yields a set of nonlinear algebraic equations, which are solved iteratively by applying Newton-Raphson's method.

\section{Results and discussion}

Figure 2 shows the variation of the scaled mobility $\mu_{E}$ as a function of double-layer thickness $\kappa a$ at various values of parameter $n$ for the case when $\lambda U / a$ is small; those for larger values of $\lambda U / a$ are presented in Figs. 3 and 4. The results for the case of Newtonian fluid $(\lambda U / a=0)$ are also presented in these figures for comparison. Figures 2-4 reveal that, for a fixed $\kappa a, \mu_{E}$ increases with the increase in $\lambda U / a$. According to Eq. (5), the larger the value of $\lambda$, the more significant the effect of shear thinning and, therefore, the greater the mobility. Figures $2-4$ also indicate that, for a fixed $\lambda U / a$, the difference between the mobility based on the present shear-thinning fluid and that based on the corresponding Newtonian fluid increases with the increase in $\kappa a$ and with the decrease in $n$. The latter is because the smaller the value of $n$, the more significant the effect of shear thinning, as suggested by Eq. (5). The former can

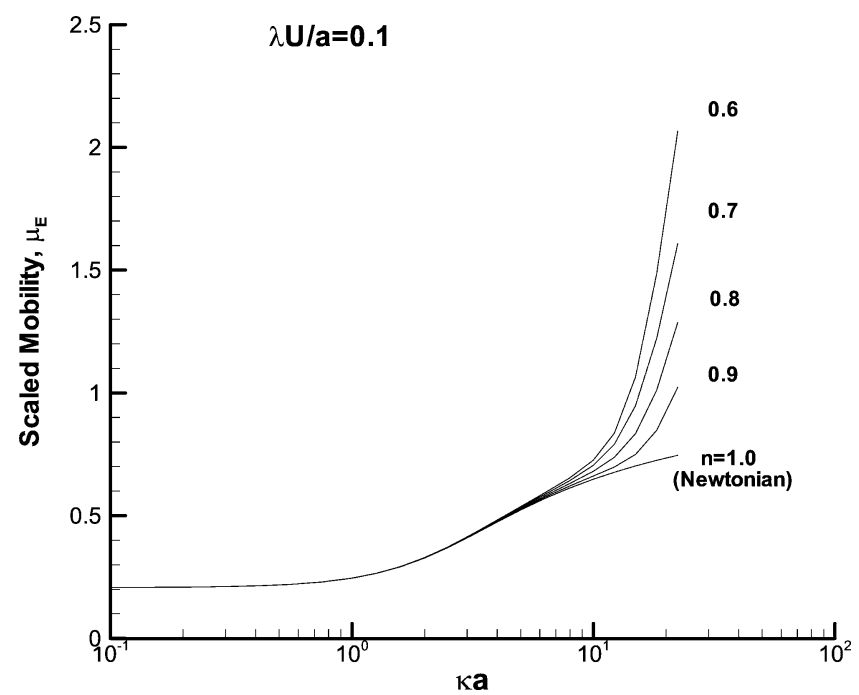

Fig. 3. Variation of scaled mobility $\mu_{E}$ as a function of double-layer thickness $\kappa a$ at various values of parameter $n$ for the case $\lambda U / a=0.1$.

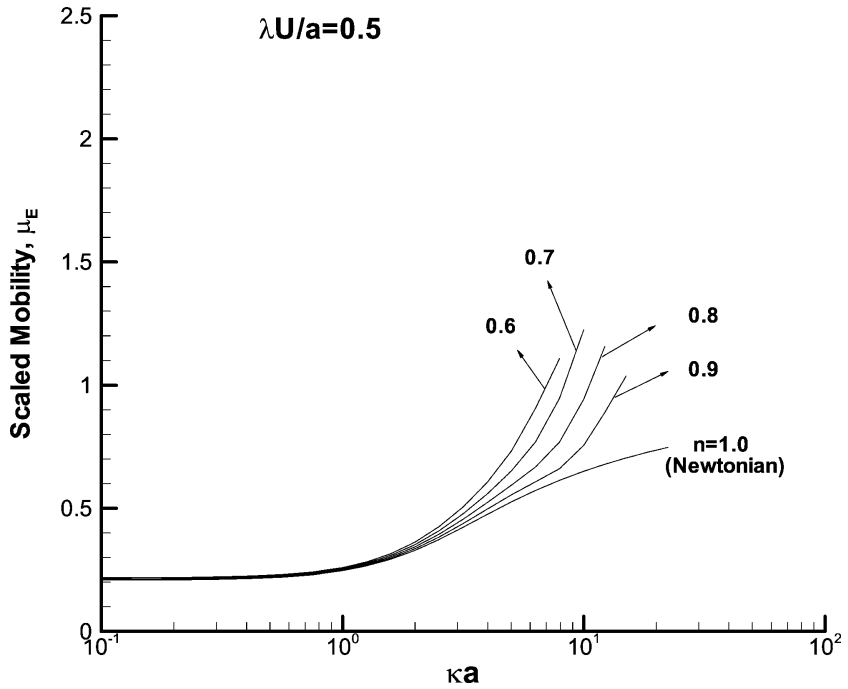

Fig. 4. Variation of scaled mobility $\mu_{E}$ as a function of double-layer thickness $\kappa a$ at various values of parameter $n$ for the case $\lambda U / a=0.5$.

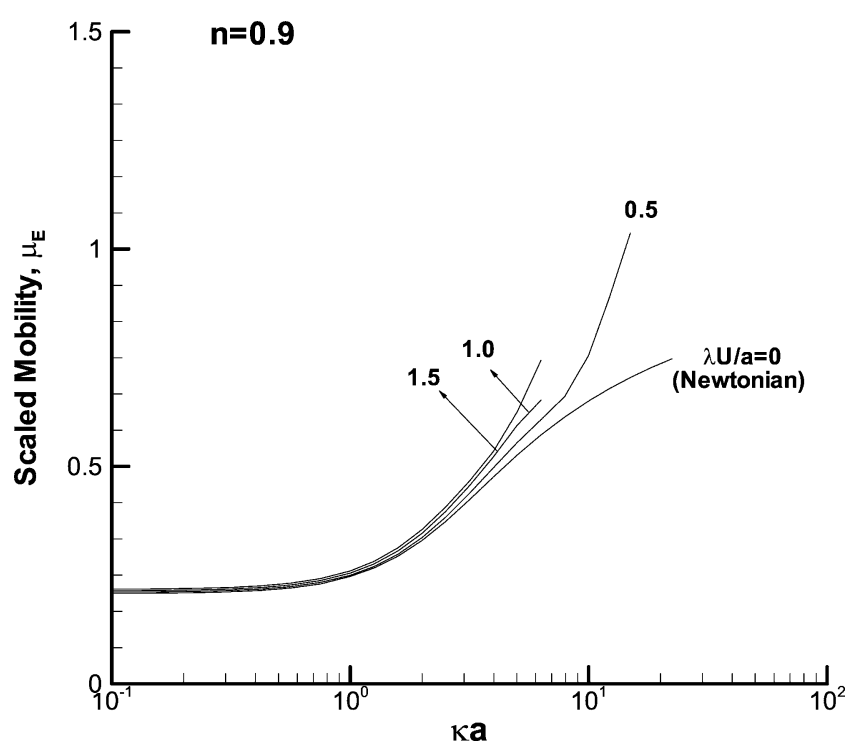

Fig. 5. Variation of scaled mobility $\mu_{E}$ as a function of double-layer thickness $\kappa a$ at various $\lambda U / a$ for the case $n=0.9$.

be explained by the following. Since no-slip condition is assumed on the particle surface, the characteristic shear rate for the present electrophoretic phenomenon can be measured by $U /(1 / \kappa)$; the larger its value, the greater the shear rate. Therefore, if the double layer is thin $(1 / \kappa$ is small or $\kappa a$ is large), the characteristic shear rate is large, and the effect of shear thinning becomes significant.

The variation of scaled mobility $\mu_{E}$ as a function of double-layer thickness $\kappa a$ at various $\lambda U / a$ for the case when $n$ is large is presented in Fig. 5, and those for smaller values of $n$ are illustrated in Figs. 6 and 7. Figure 5 reveals that both the mobility based on the present shear-thinning fluid and that based on the corresponding Newtonian fluid increase with $\kappa a$, and for a fixed $\kappa a$, the difference between them increases with $n$. These observations are consistent 


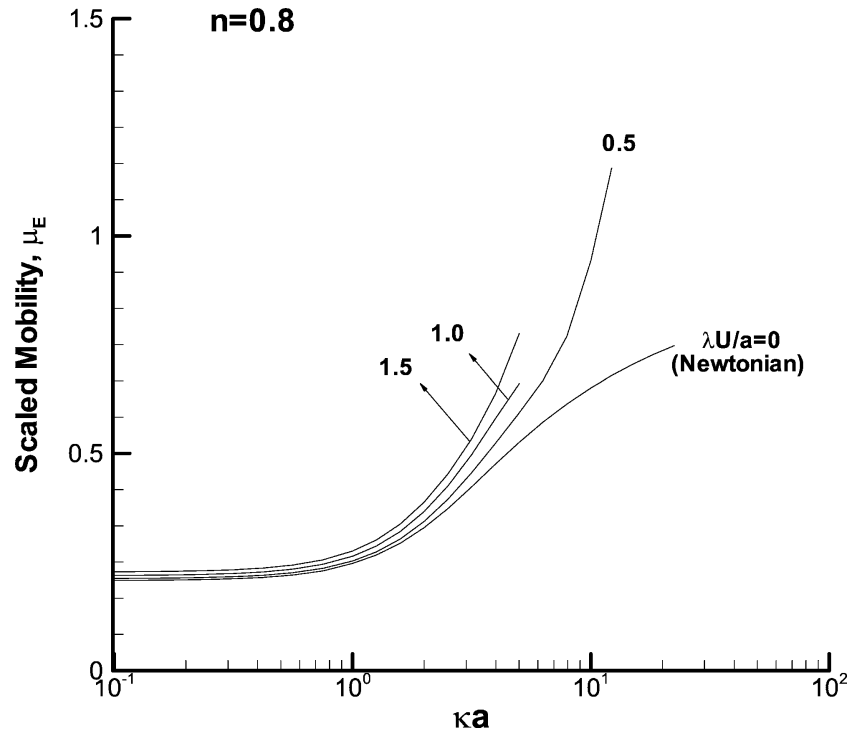

Fig. 6. Variation of scaled mobility $\mu_{E}$ as a function of double-layer thickness $\kappa a$ at various $\lambda U / a$ for the case $n=0.8$.

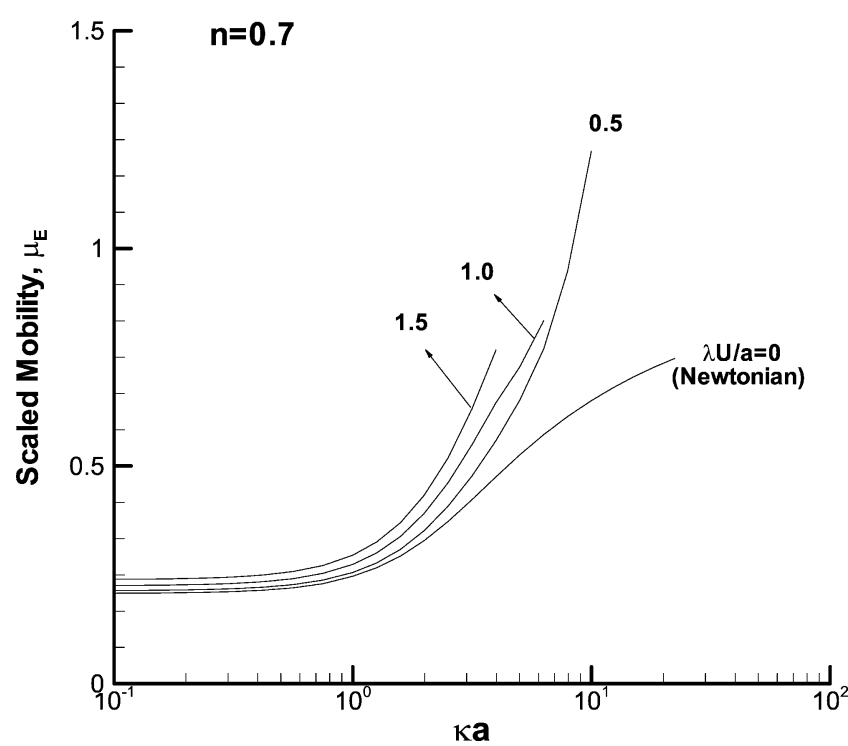

Fig. 7. Variation of scaled mobility $\mu_{E}$ as a function of double-layer thickness $\kappa a$ at various $\lambda U / a$ for the case $n=0.7$. with the results shown in Figs. 2-4. Figures 5-7 also suggest that the difference in the mobility based on the present shear-thinning fluid and that based on the corresponding Newtonian fluid increases with the decrease in the magnitude of $n$. This is expected since the smaller the $n$, the more significant the effect of shear thinning.

Figures 2-7 reveal that appreciable variation in $\mu_{E}$ occurs when $\kappa a$ is greater than unity. This is because by referring to Eq. (33), the effect of $\kappa a$ on the mobility is of a square nature. Therefore, if $\kappa a$ is smaller than unity, its influence is limited. However, as $\kappa a$ exceeds unity, its influence becomes significantly apparent.

\section{Acknowledgment}

This work is supported by the National Science Council of the Republic of China.

\section{References}

[1] M. Smoluchowski, Z. Phys. Chem. 92 (1918) 129.

[2] E. Huckel, Phys. Z. 25 (1924) 204.

[3] D.C. Henry, Proc. R. Soc. London Ser. A 133 (1931) 106.

[4] F. Booth, Proc. R. Soc. London Ser. A 203 (1950) 514.

[5] S. Levine, G.H. Neale, J. Colloid Interface Sci. 47 (1974) 520.

[6] M.W. Kozak, E.J. Davis, J. Colloid Interface Sci. 127 (1989) 497.

[7] M.W. Kozak, E.J. Davis, J. Colloid Interface Sci. 129 (1989) 166.

[8] H. Ohshima, J. Colloid Interface Sci. 188 (1997) 481.

[9] R.W. O’Brien, L.R. White, J. Chem. Soc. Faraday Trans. 274 (1978) 1607.

[10] E. Lee, J.W. Chu, J.P. Hsu, J. Colloid Interface Sci. 205 (1998) 65.

[11] E. Lee, J.W. Chu, J.P. Hsu, J. Colloid Interface Sci. 209 (1998) 240.

[12] E. Lee, F.Y. Yen, J.P. Hsu, Electrophoresis 21 (2000) 475.

[13] J.P. Hsu, E. Lee, F.Y. Yen, J. Chem. Phys. 112 (2000) 6404.

[14] R.J. Hunter, Foundations of Colloid Science, Vol. 1, Oxford Univ. Press, Oxford, 1989.

[15] R.J. Hunter, Foundations of Colloid Science, Vol. 2, Oxford Univ. Press, Oxford, 1989.

[16] R.B. Bird, R.C. Armstrong, O. Hassager, Dynamics of Polymer Liquids, Vol. 1, Wiley, New York, 1987.

[17] P.J. Carreau, Ph.D. thesis, University of Wisconsin, Madison, 1968.

[18] K. Yasuda, R.C. Armstrong, R.E. Cohen, Rheol. Acta 20 (1981) 163.

[19] C. Canuto, M.Y. Hussaini, A. Quarteroni, T.A. Zang, Spectral Methods in Fluid Dynamics, Springer-Verlag, New York, 1986. 